\title{
Perfusion imaging of the right perisylvian neural network in acute spatial neglect
}

\author{
Regine Zopf ${ }^{1}{ }^{*}$, Monika Fruhmann Berger ${ }^{1}$, Uwe Klose ${ }^{2}$ and Hans-Otto Karnath ${ }^{1}$ \\ I Section of Neuropsychology, Center of Neurology, Hertie-Institute for Clinical Brain Research, University of Tübingen, Tübingen, Germany \\ 2 Department of Diagnostic and Interventional Neuroradiology, University of Tübingen, Tübingen, Germany
}

Edited by:

Chris Rorden,

University of South Carolina, USA

Reviewed by:

Jonathan Kleinman, Johns Hopkins,

USA

Rik R. Vandenberghe,

University Hospitals Leuven, Belgium;

Katholieke Universiteit Leuven,

Belgium

${ }^{*}$ Correspondence:

Regine Zopf, Macquarie Centre for

Cognitive Science, Macquarie

University, NSW 2109, Australia.

e-mail: regine.zopf@maccs.mq.edu.au
Recent studies have suggested a tightly connected perisylvian neural network associated with spatial neglect. Here we investigated whether structural damage in one part of the network typically is accompanied with functional damage in other, structurally intact areas of this network. By combining normalized fluid-attenuated inversion-recovery (FLAIR) imaging, diffusion-weighted imaging (DWI), and perfusion-weighted imaging (PWI) we asked whether or not lesions centering on fronto-temporal regions co-occur with abnormal perfusion in structurally intact parietal cortex. With thresholds applied to delineate behaviourally relevant malperfusion of brain tissue, the analysis of normalized time-to-peak (TTP) and maximal signal reduction (MSR) perfusion maps did not reveal significant changes outside the area of structural damage. In particular, we found no abnormal perfusion in the structurally intact inferior parietal lobule (IPL) and/or the temporo-parietal junction (TPJ). The present results obtained in three consecutively admitted neglect patients with fronto-temporal lesions indicate that structural damage in one part of the right perisylvian network associated with spatial neglect does not necessarily require dysfunction by malperfusion in other, structurally intact parts of the network to provoke spatial neglect. The neural tissue in the fronto-temporal cortex appears to have an original role in processes of spatial orienting and exploration.

Keywords: spatial neglect, perfusion-weighted imaging, superior temporal cortex, parietal cortex, right perisylvian neural network, magnetic resonance imaging

\section{INTRODUCTION}

Like aphasia after left hemisphere damage, spatial neglect is the most frequent and debilitating disorder after right hemisphere stroke (Heilman et al., 1983; Karnath and Zihl, 2003). Patients with spatial neglect typically show spontaneous deviation of the head and the eyes towards the ipsilesional, right side (Fruhmann-Berger and Karnath, 2005) and disregard objects and persons located on the contralesional, left side. When searching for targets such patients concentrate their exploratory movements predominantly on the right side of space (Behrmann et al., 1997; Heilman et al., 1983; Karnath, 1994; Karnath and Zihl, 2003).

Different cortical and subcortical areas have been reported as neural correlates of spatial neglect. Cortical damage to the right inferior parietal lobule (IPL) and temporo-parietal junction (TPJ) (Heilman et al., 1983; Leibovitch et al., 1998; Mort et al., 2003; Vallar and Perani, 1986), the inferior frontal gyrus (IFG) (Heilman and Valenstein, 1972; Husain and Kennard, 1996; Samuelsson et al., 1997; Stein and Volpe, 1983) as well as the superior temporal cortex and the right insula (Buxbaum et al., 2004; Committeri et al., 2007; Corbetta et al., 2005; Karnath et al., 2001, 2004; Sarri et al., 2009) have been related to spatial neglect. Furthermore, subcortical damage to the right basal ganglia and to the thalamus has been observed with the disorder (Karnath et al., 2002; Vallar and Perani, 1986). In the latter case it has been suggested that the dysfunction of cortical areas provoked by these subcortical lesions induce the behavioural disturbance (Hillis et al., 2002, 2005; Karnath et al., 2005).
Different possibilities have been discussed to explain the varying anatomical findings. Some accounts have postulated network explanations (Corbetta et al., 2005; Karnath, 2009; Thiebaut de Schotten et al., 2005). It has been suggested that the found cortical areas might form a functional network related to spatial orienting behaviour. The existence of such a network has been supported by the finding that the frontal, parietal and temporal areas in the human brain are densely connected by several white matter pathways (Catani et al., 2007; Gharabaghi et al., 2009). These regions surround the sylvian fissure - hence this network is also known as perisylvian network (Karnath, 2009). Activation of this network in spatial orienting and exploration tasks has been shown using functional magnetic imaging (fMRI) in healthy subjects (Himmelbach et al., 2006; Hopfinger et al., 2000).

If we assume that damage to the perisylvian network causes spatial neglect, several different locations of damage or combinations thereof could be possible provoking the disorder. One possibility that has recently been investigated is that damage of white matter pathways connecting cortical areas of the perisylvian network can be associated with spatial neglect (Bartolomeo et al., 2007; He et al., 2007; Karnath et al., 2009; Mesulam, 1981; Thiebaut de Schotten et al., 2005). A further possibility is that damage to the cortical grey matter regions in the perisylvian network is the main cause for spatial neglect (Karnath, 2009; Karnath et al., 2009). Furthermore, such structural damage could lead to functional deficits in other cortical networks and thus to spatial neglect (Corbetta et al., 2005). 
The aim of the current study was to test the possibility that structural damage in one part of the perisylvian network may be accompanied with functional damage in other, structurally intact areas of this network. Especially for the acute stage of stroke, it is well-known that in addition to the morphologically damaged brain tissue further brain regions may be intact in structural magnetic resonance imaging (MRI) but malperfused and thus dysfunctional. Malperfusion has been found to be present even several days after stroke onset (Perez et al., 2006). In the malperfused areas normal supply of nutrients and oxygen to the brain is disturbed (Astrup et al., 1981; Schlaug et al., 1999), possibly due to diaschisis (Infeld et al., 1995) or to vascular dysfunction. Thus, we were interested to see whether spatial neglect typically requires malfunction of particular parts of or the entire perisylvian network, independently of where the structural damage is located within this network. Using perfusion-weighted imaging (PWI) we investigated perfusion abnormalities in patients with cortical stroke lesions showing spatial neglect. In contrast to structural MRI, PWI depicts brain regions that are structurally intact, but show abnormalities in blood supply. In particular, we asked whether lesions centering on non-parietal, i.e. superior temporal, insular, or inferior frontal regions, co-occur with abnormal perfusion in the structurally intact parietal regions IPL and/or TPJ when patients show spatial neglect.

\section{MATERIALS AND METHODS SUBJECTS}

Three spatial neglect patients with a circumscribed, ischaemic stroke in the right hemisphere consecutively admitted to the Center of Neurology were included in the study. Only patients without known prior history of stroke were included. In addition, we excluded patients if older lesions were visible on the obtained structural images. All patients gave their informed consent to participate in the study, which has been performed in accordance with the ethical standards laid down in the 1964 Declaration of Helsinki and was approved by the local ethics committee (Ethik-Kommission der Medizinischen Fakultät, Eberhards-Karls-Universität, Tübingen, Germany). Patients with subcortical lesions without cortical involvement were excluded. Since stenoses are known to produce false-positive depictions of perfusion deficits, especially in time-to-peak perfusion images (Yamada et al., 2002), we also excluded those patients with a haemodynamically relevant stenosis in the internal carotid arteries, i.e. $\geq 70 \%$, demonstrated by Doppler sonography. Demographic and clinical data of the patients are given in Table 1.

\section{CLINICAL INVESTIGATION}

Spatial neglect was diagnosed when the patient exhibited one of the typical clinical behaviours, such as spontaneous eye and head orientation towards the right (Fruhmann-Berger and Karnath, 2005), orientation towards the ipsilateral side when addressed from the front or the left, or ignoring of contralesionally located people or objects. In addition, all patients were further assessed with the following three clinical tests: the 'Letter cancellation' task, the 'Bells test' and a copying task. Neglect patients had to fulfill the criterion for spatial neglect in at least two of these tests. (i) The Letter Cancellation Task (Weintraub and Mesulam, 1985): A horizontally oriented $21 \times 29.7 \mathrm{~cm}$ sheet of paper was presented to the patients on which 60 target letters ' $\mathrm{A}$ ' are distributed amid distractors; 30 targets on the right half of the page and 30 on the left. Patients were asked to cancel all of the targets. The number of targets found is reported for the left and right side of the page. Patients were classified as suffering from spatial neglect when they omitted at least five left-sided targets. (ii) The Bells Test (Gauthier et al., 1989): The task consists of seven columns each containing five targets (bells) amid 40 distractors. Three of the seven columns ( $=15$ targets) are on the left side of a horizontally oriented $21 \times 29.7 \mathrm{~cm}$ sheet of paper, one is in the middle and three are on the right ( $=15$ targets). Again patients are asked to cancel all of the targets, and the number of targets found was reported. More than five left-sided target omissions were taken to indicate neglect. (iii) Copying Task (Johannsen and Karnath, 2004): Patients were asked to copy a complex multi-object scene consisting of four figures (a fence, a car, a house, and a tree), two in each half of a horizontally oriented $21 \times 29.7 \mathrm{~cm}$ sheet of paper. Omission of at least one of the left-sided features of each figure was scored as one point, and omission of each whole figure was scored as two points. One additional point was given when left-sided figures were drawn on the right side. The maximum score was eight. A score higher than one (i.e. $>12.5 \%$ omissions) was taken to indicate neglect. Visual field defects and pareses were assessed using standardized neurological examinations.

\section{MR IMAGING AND ANALYSIS}

For the depiction of structurally lesioned brain tissue we used diffusion-weighted imaging (DWI) imaging within the first $48 \mathrm{~h}$

Table 1 | Demographic and clinical data.

\begin{tabular}{|c|c|c|c|c|c|c|c|c|c|c|c|c|}
\hline Patient & Gender & Age & $\begin{array}{l}\text { Spatial } \\
\text { neglect }\end{array}$ & \multicolumn{2}{|c|}{$\begin{array}{c}\text { Letter } \\
\text { cancellation } \\
\text { (hits) }\end{array}$} & \multicolumn{2}{|c|}{$\begin{array}{l}\text { Bells } \\
\text { test } \\
\text { (hits) }\end{array}$} & $\begin{array}{c}\text { Copying } \\
(\% \\
\text { omitted) }\end{array}$ & $\begin{array}{l}\text { Visual } \\
\text { field } \\
\text { defect }\end{array}$ & $\begin{array}{c}\text { Paresis of } \\
\text { contra- } \\
\text { lesional side }\end{array}$ & $\begin{array}{c}\text { Lesion } \\
\text { volume } \\
\text { (\% of RH) }\end{array}$ & $\begin{array}{c}\text { Days since } \\
\text { lesion } \\
\text { (clinical exam) }\end{array}$ \\
\hline N1 & F & 39 & + & 18 & 28 & 2 & 8 & 12.5 & - & + & 6.8 & 0 \\
\hline N2 & $\mathrm{F}$ & 68 & + & 1 & 27 & 0 & 15 & 37.5 & - & + & 28.4 & 0 \\
\hline
\end{tabular}

$N$, Neglect; $F$, female; $M$, male; $R H$, right hemisphere. 
post-stroke and T2-weighted fluid-attenuated inversion-recovery (FLAIR) sequences when imaging was conducted $48 \mathrm{~h}$ or later after stroke onset (Brant-Zawadzki et al., 1996; Noguchi et al., 1997; Ricci et al., 1999; Schaefer et al., 2002). The mean time between stroke and imaging used for the present analyses was 4.7 (range 0-8) days in the neglect group. Scans were obtained on a 1.5-T echoplanar imaging (EPI) capable system (Magnetom Sonata, Siemens, Erlangen, Germany). The FLAIR sequence was acquired with 72 axial slices (thickness $1 \mathrm{~mm}$; interslice gap $1 \mathrm{~mm}$ ), a field of view (FOV) of a $192 \times 256 \mathrm{~mm}^{2}$, matrix $192 \times 256$ pixels, repetition time (TR) of $9310 \mathrm{~ms}$ and an echo time (TE) of $122 \mathrm{~ms}$. DWI was performed with a single-shot EPI spin echo sequence (TR 3200 ms; TE 87 ms; FOV $230 \times 230 \mathrm{~mm}^{2}$; matrix $128 \times 128$ pixels; slice thickness $5 \mathrm{~mm}$; gap $1 \mathrm{~mm}$; b-values of 0,500 and $1000 \mathrm{~s} / \mathrm{mm}^{2}$ ). The boundary of the lesion was delineated directly on the individual MRI image for every single transverse slice using MRIcro software (Rorden and Brett, 2000 $)^{1}$. In order to illustrate the common region of structurally lesioned brain tissue, both the scan and lesion shape were then transferred into stereotaxic space using the spatial normalization algorithm provided by SPM2 ${ }^{2}$. For determination of the transformation parameters, cost-function masking was employed (Brett et al., 2001).

Dysfunctional brain tissue due to abnormal perfusion was visualized using PWI (Belliveau et al., 1990) performed in the same scanning session as the structural scan. Fifty repetitions of perfusion-weighted EPI sequences (TR 1440 ms; TE 47 ms; FOV $230 \times 230 \mathrm{~mm}^{2}$; matrix $128 \times 128 ; 12$ axial slices; slice thickness $5 \mathrm{~mm}$; gap $1 \mathrm{~mm}$ ) were obtained with gadolinium diethyl triamineene pentaacetic acid (Gd-DTPA) bolus power injected at a rate of 3-5 ml/s. The amount of bolus used depended on the body-weight of the subject. To detect possible brain areas of dysfunction we analysed two different perfusion variables: the arrival time of blood in the brain tissue (TTP) as well as the amount of blood flow (MSR). The time-to-peak (TTP) represents the time at which the largest signal drop occurs in the signal intensity curve with respect to the first image. TTP maps are generated directly from the signal intensity

${ }^{1}$ http://www.mricro.com

${ }^{2}$ http://www.fil.ion.ucl.ac.uk/spm/ curves and do not rely on deconvoluting algorithms or the choice of adequate input functions (Calamante et al., 2002; Thijs et al., 2004). A further marker for abnormal perfusion is the amount of blood flow reaching the different regions of the brain. This parameter is depicted by the cerebral blood flow (CBF) value and the closely related maximum signal reduction (MSR) value, which also can be calculated directly from the signal intensity curve (Klose et al., 1999; Liu et al., 2002). The ratio between grey and white substance in MSR maps is 2.47:1 (Häussler et al., 1992) and thus is similar to that found for CBF. This indicates good comparability of MSR with CBF measures (Klose et al., 1999). MSR represents the extent of signal drop standardized by the baseline signal intensity (images which are recorded before contrast agent is given) (Cha et al., 2000; Klose et al., 1999; Liu et al., 2002). MSR is also designated as maximal signal drop (MSD) (Cha et al., 2000; Liu et al., 2002).

In order to identify common regions of perfusion abnormality, the PWI volumes were spatially realigned and then transferred into stereotaxic space using the spatial normalization algorithm provided by SPM2 (Figure 1). The normalized TTP and MSR maps were spatially smoothed with a Gaussian filter of $2 \mathrm{~mm}$. For SPM normalization, we used a template featuring symmetrical left-right hemispheres (cf. Aubert-Broche et al., 2003). Subsequently voxelwise inter-hemispheric comparisons were performed for each individual before extracting perfusion deficit volumes. This method takes regional biases for perfusion parameters into account, as each region is compared voxel-by-voxel to its mirrored region, thereby comparing homologous regions and avoiding a region-specific bias (cf. Karnath et al., 2005). For the normalized TTP maps, we subtracted from each voxel of the affected right hemisphere its mirrored voxel in the unaffected left hemisphere. Similarly, we divided each voxel of the affected hemisphere in the normalized MSR map by its mirrored voxel in the unaffected hemisphere. For depiction of perfusion abnormalities we defined different thresholds for TTP delays of $\geq 3 \mathrm{~s}, \geq 1.5 \mathrm{~s}$ and $\geq 0 \mathrm{~s}$ (note that these thresholds were defined on the basis of our PWI scanning time resolution), as well as for MSR fractions of $\leq 40 \%, \leq 70 \%$, and $\leq 100 \%$. For example the TTP delay of $\geq 3 \mathrm{~s}$ indicates a delay in blood arrival of $3 \mathrm{~s}$ or more and the MSR fraction of $\leq 40 \%$ indicates that the amount of blood flow is reduced to $40 \%$ or less, both compared to the homologous

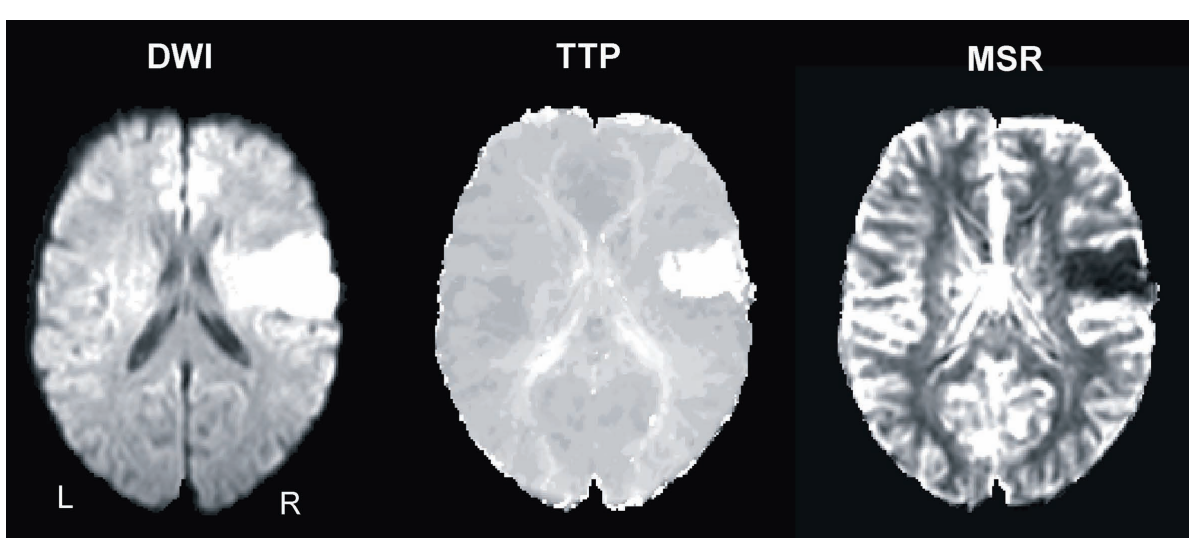

FIGURE 1 | Example from one patient's normalized diffusion-weighted imaging (DWI) as well as time-to-peak (TTP) and maximal signal reduction (MSR) perfusion maps. 
region in the left hemisphere. The resulting maps are denoted 'TTP delay maps' and 'MSR fraction maps' in the following.

\section{RESULTS}

Figure 2 presents the normalized DWI/FLAIR data. The maximum of lesion overlap for the patient group with spatial neglect centred on the superior temporal gyrus (STG), insula, IFG, pre- and postcentral gyri, and underlying white matter.

Figure 3 illustrates the overlay plots for the perfusion abnormalities of the patient group with spatial neglect. For the lowest thresholds (TTP delay $\geq 0 \mathrm{~s}$ and MSR fraction $\leq 100 \%$ ) significant overlap in many different regions is shown. This is due to the fact that with such low thresholds also regions are depicted that demonstrate no or only very small perfusion differences between the hemispheres. As the thresholds increase (TTP delay $\geq 1.5 \mathrm{~s}$ and MSR fraction $\leq 70 \%$ ), the areas of overlap became smaller. Small areas of significant overlap were found in the insula for TTP and in the STG, IFG, insula, operculum and postcentral gyrus for MSR. For the next higher thresholds (TTP delays $\geq 3$ s and MSR fraction $\leq 40 \%$ ) significant centers of overlap were no longer observed.

In order to directly compare those areas showing structural damage (Figure 2) with those showing abnormal perfusion (Figure 3), we determined the area of mismatch between DWI/
FLAIR and PWI abnormalities, i.e. the zones of structurally intact but dysfunctional neural tissue (Figure 4). For this comparison we used TTP delays $\geq 3$ s and MSR fractions $\leq 40 \%$ as thresholds. The reason was that previous work on crossed cerebellar diaschisis has shown that not all observed perfusion abnormalities are necessarily functionally relevant to the observed behaviour (Infeld et al., 1995). Previous studies have suggested that these values represent the thresholds for delineating behaviourally relevant malperfusion of brain tissue. For example, TTP delays $>2.5 \mathrm{~s}$ in Wernicke's area were associated with language dysfunction (Hillis et al., 2001), and the functional impairment of stroke patients correlated best with the volume of PWI abnormality for TTP delays $\geq 4$ s (NeumannHaefelin et al., 1999). For MSR fractions, no specific correlations between inter-hemispheric and behavioural deficits have been reported so far. However, since MSR is closely related to relative cerebral blood flow (rCBF) in stroke patients (Klose et al., 1999; Liu et al., 2002), the MSR fraction threshold was based on the observation that for $\mathrm{rCBF}$ the averaged values of $37 \%$ and $43 \%$ (relative to unaffected contralesional tissue) characterize best the penumbra, i.e. the non-functional but salvageable tissue, surrounding the infarct (Neumann-Haefelin et al., 2000; Schlaug et al., 1999). These MSR-based fraction values are also in agreement with CBF thresholds derived from positron emission tomography studies. The mean
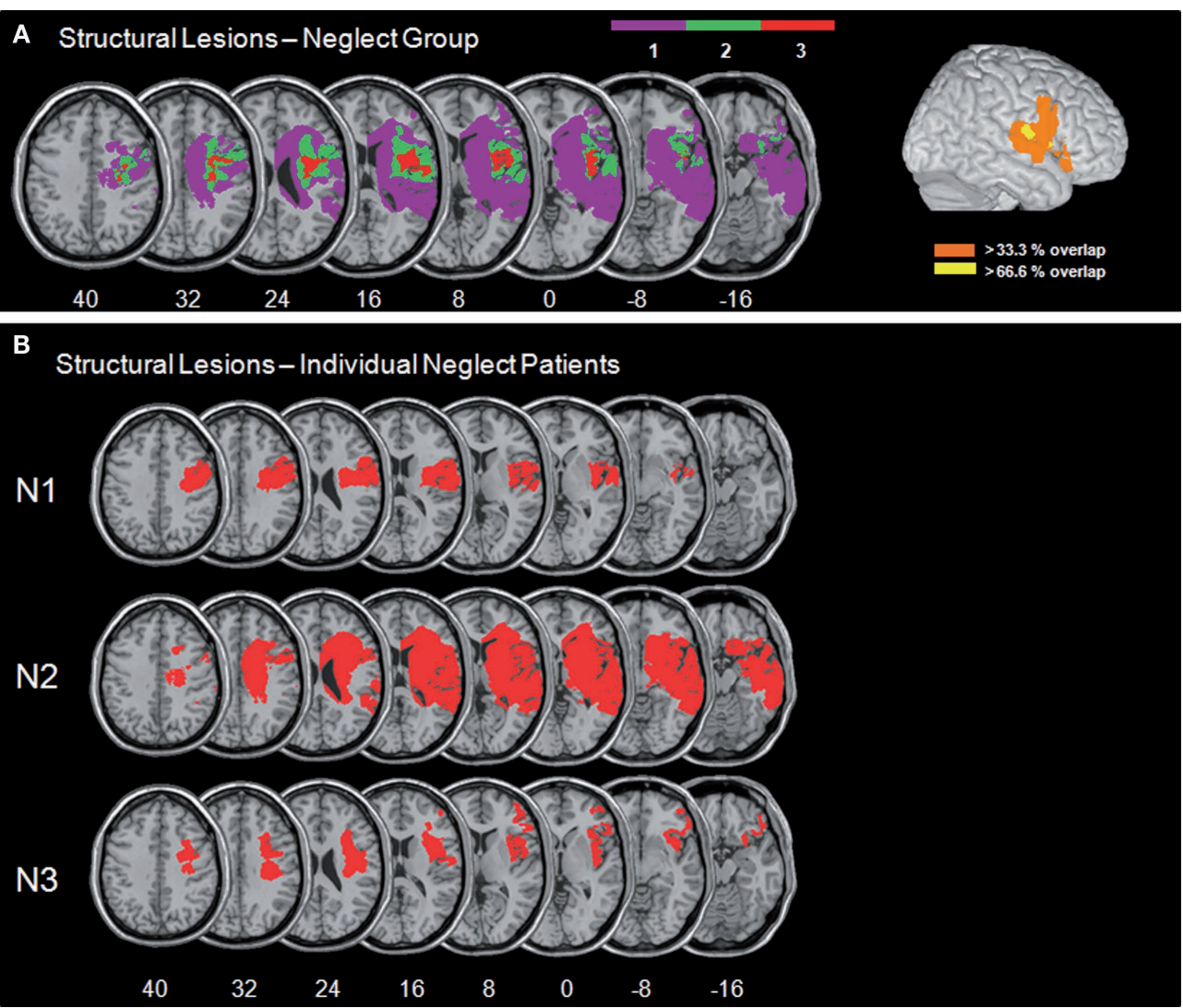

FIGURE 2 | (A) Overlay plots and lateral surface views of the normalized DWI/ FLAIR lesions for the patient group with spatial neglect. The number of overlapping areas are illustrated by different colours coding increasing frequencies from violet $(n=1)$ to red $(n=3)$. On the lateral surface view,
$>33.3 \%$ indicates that with respect to our sample at least two patients had lesioned tissue in this area and $>66.6 \%$ indicates that all patients had a lesion in this area. (B) Normalized DWI/FLAIR lesions for individual patients with spatial neglect. $\mathrm{MNI}$ z-coordinates of the transverse sections are given. 

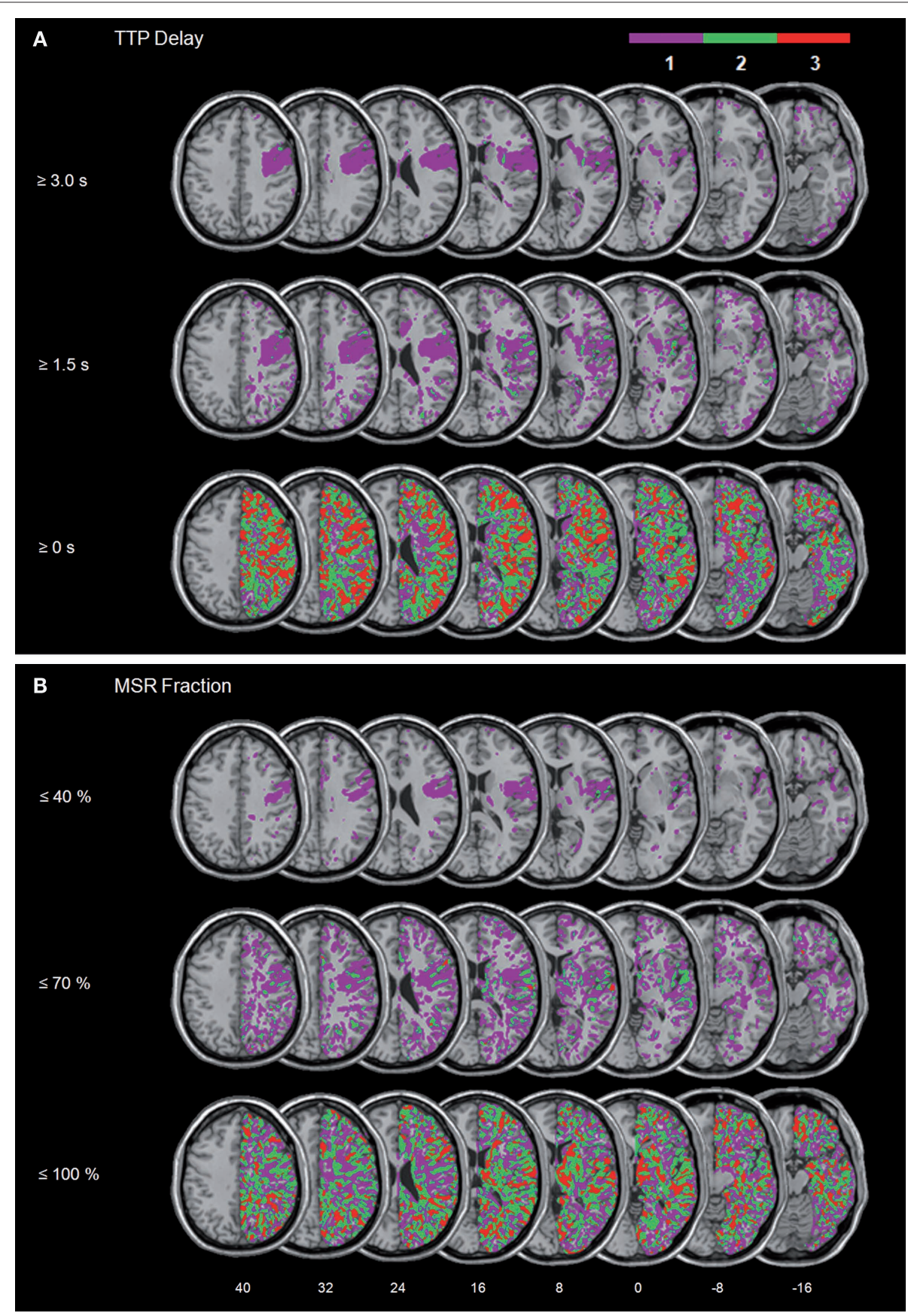

FIGURE 3 | Patient group with spatial neglect. Overlay plots of (A) the normalized maps of TTP delays $\geq 3 \mathrm{~s}, \geq 1.5 \mathrm{~s}$, and $\geq 0 \mathrm{~s}$ as well as (B) the normalized maps for MSR fractions $\leq 40 \%, \leq 70 \%$, and $\leq 100 \%$. The number of overlapping areas are illustrated by different colours coding increasing frequencies from violet $(n=1)$ to red $(n=3)$. MNI $z$-coordinates of the transverse sections are given.
$\mathrm{CBF}$ in normal brain tissue is approximately $50 \mathrm{ml} / 100 \mathrm{~g} / \mathrm{min}$, while functional impairment occurs at flow thresholds of around $20 \mathrm{ml} / 100 \mathrm{~g} / \mathrm{min}$, i.e. when the CBF is reduced to about $40 \%$ (for a review, see Baron, 2001).

For each subject we subtracted the normalized DWI/FLAIR map from (i) the normalized TTP delay map and (ii) from the normalized MSR fraction map. Figure 4 shows the superimposed mismatch maps. For the neglect patients, only few voxels in single patients (violet colour in Figure 4 indicates $n=1$ subject) were found to be malperfused though structurally intact (Figure 4).

In sum, the analysis of neglect patients' perfusion data did not reveal a systematic involvement of dysfunctional cortical brain areas in addition to the ones found to be structurally damaged. The comparison of those areas showing structural damage with those which were malperfused suggested large portions of overlap between the two. 


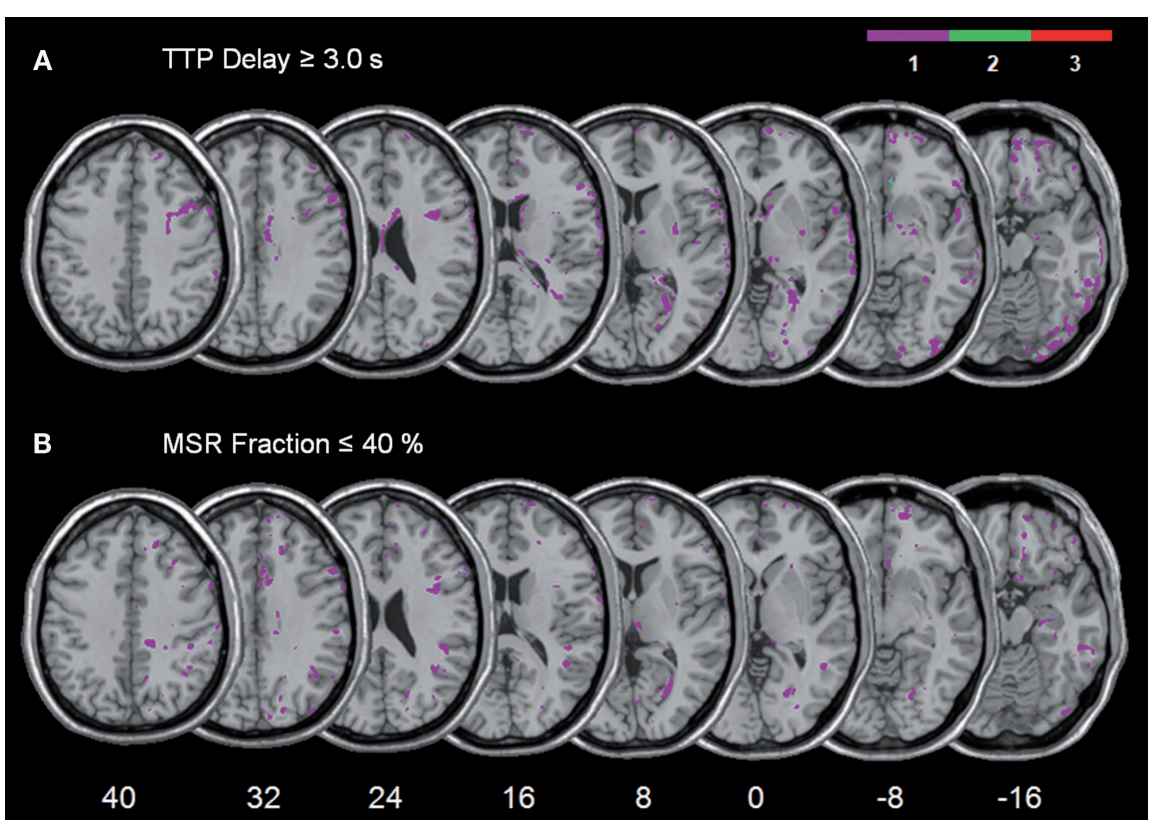

FIGURE 4 | Overlay plots showing the common regions of structurally intact but malperfused brain tissue for the patient group with spatial neglect. (A) The mismatch between TTP $\geq 3.0$ s delay maps and DWI/FLAIR. (B) The mismatch between MSR $\leq 40 \%$ fraction maps and DWI/FLAIR. The number of overlapping areas are illustrated by different colours coding increasing frequencies from violet $(n=1)$ to red $(n=3)$. MNI $z$-coordinates of the transverse sections are given.

\section{DISCUSSION}

The present study used normalized PWI to identify abnormally perfused cortical tissue in three consecutively admitted patients with spatial neglect. We investigated if structural damage in one part of the right perisylvian network associated with spatial neglect is necessarily accompanied with functional damage in other, structurally intact areas of this network. With thresholds applied to delineate behaviourally relevant malperfusion of brain tissue, we found no significant mismatch between structural lesion and abnormal perfusion in our neglect patients. The analysis of malperfused tissue as depicted by normalized TTP and MSR maps did not reveal significant perfusion changes outside the area of structural damage.

Stroke patients with perfusion deficits that were mainly matched by or were smaller than the actual structural lesion also were observed in a study by Restrepo et al. (2005). These authors investigated the aetiology of brain ischemia in 130 patients using DWI and PWI. Restrepo and co-workers found perfusion abnormalities which were smaller than ('negative' DWI/PWI mismatch) or matched the region of structural damage in $51 \%$ of the patients. A mismatch with a larger area of malperfusion compared to the DWI lesions ('positive' DWI/PWI mismatch) was noted in the remaining portion of the sample. Restrepo et al. (2005) found that a 'positive' mismatch was associated with 'large-artery atherosclerosis'. The latter was defined as stenosis of a major (intra- or extracranial) artery $>50 \%$. Large-artery atherosclerosis increased the odds of a positive mismatch by a factor of four, indicating that malperfusion which extends the area of structural damage is likely to occur with a major artery stenosis $>50 \%$. Also, Yamada et al. (2002) observed that haemodynamically relevant stenoses of the intracerebral artery (ICA) produce depictions of perfusion deficits, especially in TTP perfusion images.
To avoid false-positive depictions of abnormal cortical perfusion due to ICA stenoses rather than due to the cortical strokes, the present study excluded patients with haemodynamically relevant stenoses of the ICA. Under these conditions we found that structural damage centering on non-parietal, i.e. superior temporal, insular, or inferior frontal regions, is not necessarily accompanied by significant perfusion deficits outside of the lesion territory. In particular, we found no significant abnormal perfusion in the structurally intact parietal regions IPL and/or TPJ. Structural damage in one part of the perisylvian network obviously does not necessarily require dysfunction by malperfusion in other, structurally intact parts of the network to provoke spatial neglect. The neural tissue in the fronto-temporal cortex appears to have an original role in processes of spatial orienting and exploration.

Obviously, this is systematically different for subcortical lesions. In clear contrast to our present findings on cortical stroke, patients with spatial neglect following subcortical lesions showed large perfusion deficits in structurally intact cortical areas of the perisylvian network, while subcortical stroke patients without neglect did not show such perfusion abnormalities (Hillis et al., 2002, 2005; Karnath et al., 2005). These findings suggested that spatial neglect due to subcortical stroke is evoked by dysfunction of structurally intact cortical areas rather than through the neuronal loss in the subcortical structures itself.

We would like to point out that our present conclusions are based on a small sample size. Therefore, we do not intend to exclude the possibility that a cortical lesion combined with a large perfusion deficit extending the borders of the lesion territory may also be observed in association with cortical spatial neglect. Nevertheless, the present cases of consecutively admitted patients with spatial neglect and fronto-temporal lesions demonstrate at least that 
such additional malperfusion in structurally intact regions of the perisylvian network is not an absolutely required condition if patients show spatial neglect after a cortical lesion. Nevertheless, future studies are necessary to further investigate this conclusion.

PWI is used to assess abnormalities in blood supply taskindependently. It has been shown that the size and location of the tissue with abnormal perfusion identified by PWI correlates well with stroke patients' behavioural dysfunction (Baird et al., 2000; Barber et al., 1998; Beaulieu et al., 1999; Hillis et al., 2001; Neumann-Haefelin et al., 1999; Tong et al., 1998). In contrast to PWI, BOLD activation in fMRI is mainly studied in combination with a specific task. Thus, it may well be that although we found no perfusion deficits in areas outside the region of structural damage such areas could still show specific, task-dependent functional deficits. For example, Corbetta et al. (2005) used a Posner-like covert attentional shifting task in patients with lesions centering on the TPJ, STG, insula, frontal operculum, putamen and white matter. They observed that spatial neglect as well as recovery of spatial neglect was associated with task-related disturbances and restorations of the BOLD signal in ventral temporo-parietal and

\section{REFERENCES}

Astrup, J., Siesjö, B. K., and Symon, L. (1981). Thresholds in cerebral ischemia - the ischemic penumbra. Stroke 12, 723-725.

Aubert-Broche, B., Grova, C., Jannin, P., Buvat, I., Benali, H., and Gibaud, B. (2003). Detection of interhemispheric asymmetries of brain perfusion in SPECT. Phys. Med. Biol. 48, 1505-1517.

Baird, A. E., Lövblad, K.-O., Dashe, J. F., Connor,A., Burzynski, C., Schlaug, G., Straroselskaya, I., Edelman, R. R., and Warach, S. (2000). Clinical correlations of diffusion and perfusion lesion volumes in acute ischemic stroke. Cerebrovasc. Dis. 10, 441-448.

Barber,P.A., Darby, D. G., Desmond, P. M., Yang, Q., Gerraty, R. P., Jolley, D., Donnan, G. A., Tress, B. M., and Davis, S. M. (1998). Prediction of stroke outcome with echoplanar perfusion- and diffusion-weighted MRI. Neurology 51, 418-426.

Baron, J.-C. (2001). Perfusion thresholds in human cerebral ischemia: historical perspective and therapeutic implications. Cerebrovasc. Dis. 11, 2-8.

Bartolomeo, P., Thiebaut de Schotten, M., and Doricchi, F. (2007). Left unilateral neglect as a disconnection syndrome. Cereb. Cortex 17, 2479-2490.

Beaulieu, C., de Crespigny, A., Tong, D. C., Moseley, M. E., Albers, G. W., and Marks, M. P. (1999). Longitudinal magnetic resonance imaging study of perfusion and diffusion in stroke: evolution of lesion volume and correlation with clinical outcome. Ann. Neurol. 46, 568-578.

Behrmann, M., Watt, S., Black, S. E., and Barton, J. J. (1997). Impaired visual search in patients with unilateral neglect: an oculographic analysis. Neuropsychologia 35, 1445-1458.

Belliveau, J.W., Rosen, B. R., Kantor, H. L., Rzedzian, R. R., Kennedy, D. N., McKinstry, R. C., Vevea, J. M., Cohen, M.S., Pykett, I.L., and Brady, T. J. (1990). Functional cerebral imaging by susceptibilitycontrast NMR. Magn. Reson. Med. 14, 538-546.

Brant-Zawadzki, M., Atkinson, D., Detrick, M., Bradley, W. G., and Scidmore, G. (1996). Fluid-attenuated inversion-recovery (FLAIR) for assessment of cerebral infarction. Initial clinical experience in 50 patients. Stroke 27, 1187-1191.

Brett, M., Leff, A. P., Rorden, C., and Ashburner, J. (2001). Spatial normalization of brain images with focal lesions using cost function masking. Neuroimage 14, 486-500.

Buxbaum, L. J., Ferraro, M. K., Veramonti, T., Farne, A., Whyte, J., Coslett, H.B. (2004). Hemispatial neglect: subtypes, neuranatomy and disability. Neurology 62, 749-756.

Calamante, F., Gadian, D. G., and Connelly, A. (2002). Quantification of perfusion using bolus tracking Assumptions, limitations, and potential implications for clinical use. Stroke 33, 1146-1151.

Catani, M., Allin, M. P. G., Husain, M., Pugliese, L., Mesulam, M.-M., Murray, R. M., and Jones, D. K. (2007). Symmetries in human brain language pathways correlate with verbal recall. Proc. Natl. Acad. Sci. U.S.A. 104, 17163-17168. Ladavas, E., Frassinetti, F., and magnetic resonance imaging in stroke.

dorsal parietal areas. However, it has also been shown that in stroke patients the BOLD signal can be abnormal in brain regions without functional deficits (Rossini et al., 2004).

In conclusion, we observed that structural damage in one part of the right perisylvian network associated with spatial neglect does not necessarily require dysfunction by malperfusion in other, structurally intact parts of the network to provoke spatial neglect. Lesions centering on superior temporal, insular, and inferior frontal regions were not accompanied by significant perfusion deficits outside of the lesion territory, particularly not in the intact IPL and/or TPJ. Future studies are required to specifically look at structural damage to the grey and white matter as well as at task-dependent and task-independent functional deficits in subjects with cortical strokes at different stages following stroke-onset.

\section{ACKNOWLEDGEMENTS}

This work was supported by the Bundesministerium für Bildung und Forschung (BMBF-Verbundprojekt "Räumliche Orientierung" 01GW0641) and the Deutsche Forschungsgemeinschaft (SFB 550-A4).

Cha, S., Lu, S., Johnson, G., and Knopp, E. A. (2000). Dynamic susceptibility contrast MR imaging: correlation of signal intensity changes with cerebral blood volume measurements. J. Magn. Reson. Imaging 11, 114-119.

Committeri, G., Pitzalis, S., Galati, G., Patria, F., Pelle, G., Sabatini, U. Castriota-Scanderbeg, A., Piccardi, L. Guariglia, C., and Pizzamiglio, L. (2007). Neural base of personal and extrapersonal neglect in humans. Brain 130, 431-441.

Corbetta, M., Kincade, M. J., Lewis, C. Snyder, A. Z., and Sapir, A. (2005). Neural basis and recovery of spatial attention deficits in spatial neglect. Nat. Neurosci. 8, 1603-1610.

Fruhmann-Berger,M., and Karnath, H.-O. (2005). Spontaneous eye and head position in patients with spatial neglect. J. Neurol. 10, 1194-1200.

Gauthier, L., Dehaut, F., and Joanette, Y. (1989). The bells test: a quantitative and qualitative test for visual neglect. Int. J. Clin. Neuropsychol. 11, 49-54.

Gharabaghi,A., Kunath, F.,Erb, M.,Saur, R. Heckl, M., Tatagiba, M., Grodd, W., and Karnath,H.O. (2009). Perisylvian white matter connectivity in the human right hemisphere. BMC Neurosci. 10, 15.

Häussler, M.D.,Wallner, B., and Bargon, G. (1992). Darstellung regionaler Hirnperfusion mit dynamischer Ma gnetresonanztomographie. Fortschr. Röntgenstr. 156, 207-211.

He, B. J., Snyder, A. Z., Vincent, J. L., Epstein, A., Shulman, G. L., and Corbetta, M. (2007). Breakdown of functional connectivity in frontoparietal networks underlies behavioral deficits in spatial neglect. Neuron 53, 905-918.
Heilman, K. M., and Valenstein, E. (1972). Frontal lobe neglect in man. Neurology 22, 660-664.

Heilman, K. M., Watson, R. T., Valenstein, E., and Damasio, A. R (1983). Localization of lesions in neglect. In Localization in Neuropsychology, A. Kertesz, ed. (New York, Academic Press), pp. 471-492.

Hillis, A. E., Newhart, M., Heidler, J., Barker, P. B., Herskovits, E. H., and Degaonkar, M. (2005). Anatomy of spatial attention: insights from perfusion imaging and hemispatial neglect in acute stroke. J. Neurosci. 25, 3161-3167.

Hillis, A. E., Wityk, R. J., Barker, P. B., Beauchamp, N. J., Gailloud, P., Murphy, K., Cooper, O., and Metter, E. J. (2002). Subcortical aphasia and neglect in acute stroke: the role of cortical hyperfusion. Brain 125, 1094-1104.

Hillis, A. E., Wityk, R. J., Tuffiash, E., Beauchamp, N. J., Jacobs, M.A., Barker, P. B., and Selnes, O. A. (2001). Hypoperfusion of Wernicke's area predicts severity of semantic deficit in acute stroke. Ann. Neurol. 50, 561-566.

Himmelbach, M., Erb, M., and Karnath, H. O. (2006). Exploring the visual world: the neural substrate of spatial orienting. Neuroimage 32, 1747-1759.

Hopfinger, J. B., Buonocore, M. H., and Mangun, G. R. (2000). The neural mechanisms of top-down attentional control. Nat. Neurosci. 3, 284-291.

Husain, M., and Kennard, C. (1996). Visual neglect associated with frontal lobe infarction. J. Neurol. 243, 652-657. 
Infeld, B., Davis, S. M., Lichtenstein, M. Mitchell, P. J., and Hopper, J. L. (1995). Crossed cerebellar diaschisis and brain recovery after stroke. Stroke 26, 90-95.

Johannsen, L., and Karnath, H.-O. (2004). How efficient is a simple copying task to diagnose spatial neglect in its chronic phase. J. Clin. Exp. Neuropsychol. 26, 251-256.

Karnath,H.-O.(1994).Spatiallimitation of eye movements during ocular exploration of simple line drawings in neglect syndrome. Cortex 30, 319-330.

Karnath,H.-O. (2009). A right perisylvian neural network for human spatial orienting. In The Cognitive Neurosciences IV, M. S. Gazzaniga, ed. (Cambridge, MA, MIT Press), pp. 259-268.

Karnath, H.-O., Ferber, S., and Himmelbach, M. (2001). Spatial awareness is a function of the temporal not the posterior parietal lobe. Nature 411, 950-953.

Karnath, H.-O., Fruhmann Berger, M., Küker, W., and Rorden, C. (2004). The anatomy of spatial neglect based on voxelwise statistical analysis: a study of 140 patients. Cereb. Cortex 14, 1164-1172.

Karnath, H.-O., Fruhmann Berger, M., Zopf, R., and Kuker, W. (2004). Using SPM normalization for lesion analysis in spatial neglect. Brain 127(Pt4), E10; author reply E11.

Karnath, H.-O., Himmelbach, M., and Rorden, C. (2002). The subcortical anatomy of human spatial neglect: putamen, caudate nucleus, and pulvinar. Brain 125, 350-360.

Karnath,H. O., Rorden, C., and Ticini, L. F. (2009). Damage to white matter fiber tracts in acute spatial neglect. Cereb. Cortex. doi: 10.1093/cercor/bhn1250.

Karnath, H.-O., and Zihl, J. (2003). Disorders of spatial orientation. In Neurological Disorders: Course and Treatment, 2nd Edn, T. Brandt, L. R. Caplan, J. Dichgans, H. C. Diener and C. Kennard, eds (San Diego, CA, Academic Press), pp. 277-286.

Karnath, H.-O., Zopf, R., Johannsen, L., Fruhmann Berger, M., Nägele, T., and Klose, U. (2005). Normalized perfusion MRI to identify common areas of dysfunction: patients with basal ganglia neglect. Brain 128, 2462-2469.

Klose, U., Nägele, T., Friese, S., and Bitzer, M. (1999). Charakteristische
Größen bei der MR-Untersuchung der zerebralen Durchblutung mit hoher räumlicher und zeitlicher Auflösung. Fortschr. Röntgenstr. 170, 474-481.

Leibovitch, F.S., Black, S.E., Caldwell, C. B., Ebert, P.L., Ehrlich, L. E., and Szalai, J. P. (1998). Brain-behavior correlations in hemispatial neglect using CT and SPECT. The Sunnybrook Stroke Study. Neurology 50, 901-908.

Liu, Y.-J., Chung, H.-W., Huang, I.-J., Wang, F.-N., Chin, S.-C., Lee, C.-S., and Chen, C.-Y. (2002). A reinvestigation of maximal signal drop in dynamic susceptibility contrast magnetic resonance imaging. J. Neuroimaging 12, 330-338.

Mesulam, M.-M. (1981). A cortical network to directed attention and unilateral neglect. Ann. Neurol. 10, 309-325.

Mort, D. J., Malhotra, P., Mannan, S. K., Rorden, C., Pambakian, A., Kennard, C., and Husain, M. (2003). The anatomy of visual neglect. Brain 126(Pt 9), 1986-1997.

Neumann-Haefelin, T., Wittsack, H.-J., Fink, G. R., Wenserski, F., Li, T.-Q., Seitz, R. J., Siebler, M., Mödder, U., and Freund, H.-J. (2000). Diffusion- and perfusion-weighted MRI. Influence of severe carotid artery stenosis on the DWI/PWI mismatch in acute stroke. Stroke 31, 1311-1317.

Neumann-Haefelin, T., Wittsack, H.-J., Wenserski, F., Siebler, M., Seitz, R. J., Mödder, U., and Freund, H.-J. (1999). Diffusion- and perfusion-weighted MRI. The DWI/PWI mismatch region in acute stroke. Stroke 30, 1591-1597.

Noguchi, K., Ogawa, T., Inugami, A., Fujita, H.,Hatazawa,J.,Shimosegawa, E., Okudera, T., Uemura, K., and Seto, H. (1997). MRI of acute cerebral infarction: a comparison of FLAIR and T2-weighted fast spin-echo imaging. Neuroradiology 39, 406-410.

Perez, A., Restrepo, L., Kleinman, J. T., Barker, P., Beauchamp, N., and Wityk, R. J. (2006). Patients with diffusion-perfusion mismatch on magnetic resonance imaging 48 hours or more after stroke symptom onset: clinical and imaging features. J. Neuroimaging 16, 329-333.

Restrepo, L., Jacobs, M. A., Barker, P. B., Beauchamp, N. J., Sholasky, R. L.
Keswani, S. C., and Wityk, R. J. (2005). Etiology of perfusion-diffusion MRI mismatch patterns. J. Neuroimaging 11, 1-7.

Ricci, P.E., Burdette, J.H., Elster, A. D., and Reboussin, D. M. (1999). A comparison of fast spin-echo, fluid-attenuated inversion-recovery, and diffusionweighted MR imaging in the first 10 days after cerebral infarction. Am. J. Neuroradiol. 20, 1535-1542.

Rorden, C., and Brett, M. (2000). Stereotaxic display of brain lesions. Behav. Neurol. 12, 1535-1542.

Rossini, P. M., Altamura, C., Ferretti, A. Vernieri, F., Zappasodi, F., Caulo, M. Pizzella, V., Del Gratta, C., Romani, G. L., and Tecchio, F. (2004) Does cerebrovascular disease affect the coupling between neuronal activity and local haemodynamics? Brain 127(Pt 1), 99-110.

Samuelsson, H., Jensen, C., Ekholm, S. Naver, H., and Blomstrand, C. (1997). Anatomical and neurological correlates of acute and chronic visuospatial neglect following right hemisphere stroke. Cortex 33, 271-285.

Sarri, M., Greenwood, R., Kalra, L., and Driver, J. (2009). Task-related modulation of visual neglect in cancellation tasks. Neuropsychologia 47, 91-103.

Schaefer, P. W., Hunter, G. J., He, J. Hamberg, L. M., Sorensen, A. G. Schwamm, L. H., Koroshetz, W. J., and Gonzalez, R. G. (2002). Predicting cerebral ischemic infarct volume with diffusion and perfusion MR imaging. Am. J. Neuroradiol. 23, 1785-1794.

Schlaug, G., Benfield, A., Baird, A. E., Siewert, B., Lövblad, K.-O., Parker, R. A., Edelman, R. R., and Warach, S. (1999). The ischemic penumbra. Operationally defined by diffusion and perfusion MRI. Neurology 53, 1528-1537.

Stein, S., and Volpe, B. T. (1983). Classical "parietal" neglect syndrome after subcortical right frontal lobe infarction. Neurology 33, 797-799.

Thiebaut de Schotten, M., Urbanski, M. Duffau, H., Volle, E., Levy, R., Dubois, B., and Bartolomeo, P. (2005). Direct evidence for a parietal-frontal pathway subserving spatial awareness in humans. Science 309, 2226-2228.

Thijs, V. N., Somford, D. M., Bammer, R. Robberecht, W., Moseley, M. E., and
Albers, G. W. (2004). Influence of arterial input function on hypoperfusion volumes measured with perfusion-weighted imaging. Stroke 35, 94-98.

Tong, D. C., Yenari, M. A., Albers, G. W., O'Brien, M., Marks, M. P., and Moseley, M. E. (1998). Correlation of perfusion- and diffusion-weighted MRI with NIHSS score in acute ( $<6.5$ hour) ischemic stroke. Neurology 50, 864-870.

Vallar, G., and Perani, D. (1986). The anatomy of unilateral neglect after right-hemisphere stroke lesions. A clinical/CT-scan correlation study in man. Neuropsychologia 24, 609-622.

Weintraub, S., and Mesulam, M.-M. (1985). Mental state assessment of young and elderly adults in behavioral neurology. In Principles of Behavioral Neurology, M.-M. Mesulam, ed. (Philadelphia, F.A. Davis Company), pp. 71-123.

Yamada, K., Wu, O., Gonzalez, R. G. Bakker, D., Ostergard, L., Copen, W. A., Weisskoff, R. M., Rosen, B. R., Yagi, K. Nishimura, T., and Sorensen, A. G. (2002).Magnetic resonance perfusionweighted imaging of acute cerebral infarction. Effect of the calculation methods and underlying vasculopathy. Stroke 33, 87-94.

Conflict of Interest Statement: We declare that the research was conducted in the absence of any commercial or financial relationships that could be construed as a potential conflict of interest.

Received:07 February 2009; paper pending published: 07 April 2009; accepted: 15 July 2009; published online: 03 August 2009.

Citation: Zopf R, Fruhmann Berger M, Klose U and Karnath H-O (2009) Perfusion imaging of the right perisylvian neural network in acute spatial neglect. Front. Hum. Neurosci. 3:15. doi: 10.3389/neuro.09.015.2009

Copyright (C) 2009Zopf, Fruhmann Berger, Klose and Karnath. This is an open-access article subject to an exclusive license agreement between the authors and the Frontiers Research Foundation, which permits unrestricted use, distribution, and reproduction in any medium, provided the original authors and source are credited. 